\title{
Stimulus selection and meaningfulness following a single opportunity to rehearse each paired associate
}

\author{
FRANKLIN M. BERRY, DONALD A. SHERROD \\ and LARRY E. LOVE \\ Columbus College, Columbus, Ga. 31907
}

Stimulus selection was investigated as a function of stimulus meaningfulness $(\mathrm{M})$ and list input position following a single study trial of paired-associate (PA) learning. Large individual differences were observed in Ss' cue processing strategies: only 16 of 30 Ss appeared to have developed the strategy of selecting higher $M$ words from word-trigram compound stimuli following a single exposure of each PA. There also was no systematic relationship between list input position and the occurrence of word selection for these $16 \mathrm{Ss}$. But, on the average, less than $10 \mathrm{sec}$ were required for most of the word-selecting Ss to develop the strategy of encoding the more meaningful cues for PA learning.

A number of experiments in recent years have been concerned with the relationship between stimulus selection and meaningfulness (M) in paired-associate (PA) learning. College Ss have been shown to select higher $\mathrm{M}$ colors from color-trigram stimulus compounds (e.g., Underwood, Ham, \& Ekstrand, 1962) and higher M words from word-trigram stimulus compounds (e.g., Spear, Ekstrand, \& Underwood, 1964). Some of the more recent work on this problem has been distinguished by the incorporation of degree of learning as a parameter in such studies. The rationale has been to map the development of the strategy of processing higher $\mathbf{M}$ cues during PA learning (e.g., Berry, Jobert, \& Baumeister, 1972; James \& Greeno, 1968). These studies have indicated that college Ss tend to rely almost exclusively on higher $M$ word cues from the initial trial of PA learning through list mastery; processing of trigrams apparently occurs only during overlearning trials.

The present experiment was also concerned with mapping the development of word-selection strategies by college Ss. It was designed to explore systematically the phenomenon of stimulus selection on the basis of $M$ following a single PA trial. More specifically, stimulus selection was examined following a single opportunity to rehearse ("store") each PA, i.e., after a single "study" trial, with item input position included as a parameter.

\section{METHOD \\ Subjects}

The Ss were 30 male and female Columbus College undergraduates. All Ss were volunteers, and they were assigned randomly to treatments by means of a random number table (i.e., all data protocols were randomized prior to the beginning of the experiment).

\section{Apparatus and Materials}

A Lafayette memory drum (Model 303C) was used to present the redundant-stimulus PAs. The 10-pair list of word-trigram:digit PAs was that used by Berry et al (1972). The words were presented to the left of the trigrams for one-half of the pairs, and the reverse was true for the other half. The digit response was always presented to the right of the word-trigram stimulus compound.

\section{Procedure}

A single study trial was available to each $\mathrm{S}$. During this trial, each individual pair was exposed for 2 sec. Ten different orders were used equally often to present the PAs; these orders were determined by means of a Latin-square counterbalancing procedure which ensured that each of the 10 pairs occurred once in each of the 10 possible serial positions. Each list order was used three times. Within-Ss stimulus-selection tests were presented immediately after the last PA was exposed. The words and trigrams were presented separately-all of the word cues (trigram cues) were listed on a single sheet of paper with a "blank" opposite each one. The order of presenting the words (trigrams) on the transfer test sheet was random, with the restriction that no duplication of orders used elsewhere in the experiment was permitted. Each $S$ was instructed to write the appropriate numeral in the blank opposite each word (trigram); Ss were not, however, required to guess a digit in cases of uncertainty. The order of presentation of the word-cue and trigram-cue transfer tasks was counterbalanced across Ss.

\section{RESULTS AND DISCUSSION}

Eighteen of the $30 \mathrm{Ss}$ achieved at least one correct response in the word and/or trigram transfer tests. The 12 other Ss apparently did not develop a successful PA acquisition strategy, and they are ignored in all subsequent analyses. The $18 \mathrm{Ss}$ who apparently did develop a PA acquisition strategy achieved more correct responses to the words than to the trigrams, the respective means and SDs (within parentheses) being 2.1 (1.4) and .5 (.7). As only 7 Ss achieved any correct responses to the trigrams, these means were contrasted by means of the Wilcoxon matched-pairs signed-ranks test (Siegel, 1956, p. 75). The obtained $\mathrm{T}(\mathrm{N}=17)$ was $14.0, \mathrm{p}<.01$, for a two-tailed test. Thus, in support of previous findings by Berry et al (1972), these college Ss apparently processed the words as their functional cues and largely ignored the trigrams.

The effect of input position on word selection was examined only for those Ss who achieved at least one correct response to a word cue $(\mathrm{N}=16)$. (As there was no evidence for trigram selection, the trigram data were ignored.) The mean numbers of correct responses 
achieved to word cues which had been presented in Serial Positions $1-10$ were, respectively, $.3, .3, .3, .2, .1$, $.3, .5$. As these data represent a dichotomous variable (each S was tested with one word at each serial position such that the only scores possible were 0 or 1 ), Cochran's Q statistic (Siegel, 1956, p. 161) was used to contrast performance at the various serial positions. The $\mathrm{Q}(9)$ was $10.7, \mathrm{p}>.05$. Individual differences seemed to have been more important than input position for determining the point at which Ss began processing the words from word-trigram compounds during the single study trial of PA learning.

Although no systematic input-position effect was observed in the word-selection data, it was possible to obtain an estimate of the average number of PA items that word-selecting Ss $(N=16)$ inspected before they developed the strategy of stimulus selection on the basis of M. Calculation of this average estimate was based on the assumption that the point at which $S$ had achieved the strategy of selecting words was indicated by the first PA learning input position to yield a correct response to a word cue in transfer. For example, if an $\mathrm{S}$ achieved a correct response to three different words during transfer and if these words had been presented in the fourth, fifth, and sixth serial positions during PA learning, then Serial Position (input position) 4 would be taken to be the point at which $S$ had developed the word-selection strategy. The average number of item exposures required for word-selecting Ss to begin encoding the stimuli on the basis of $\mathrm{M}$ was 4.6 with a SD of 3.3 ; the median number of item exposures was 3.5 and the modal number 1.0. These data make it clear that, if a word-selection strategy is developed during the initial PA study trial, it is developed rapidly.

The rapid development of the $M$ processing strategy (for 16 of $30 \mathrm{Ss}$ ) can be seen in another way by multiplying the average number of item exposures by the exposure duration per item. The resulting estimates are $(3.5 \times 2.0) 7.0 \mathrm{sec}$ (based on the median) and ( $4.6 \mathrm{x}$ 2.0) $9.2 \mathrm{sec}$ (based on the mean). Thus, the strategy of stimulus selection on the basis of $\mathrm{M}$ developed in less than $10 \mathrm{sec}$ of cumulative exposure to word-trigram:digit PAs. The present results also make it obvious that further research is needed to account for the role of individual differences in the acquisition of stimulus-encoding strategies. Use of a correlational research design may provide some information about the particular $S$ abilities related to the development of stimulus-selection strategies based on stimulus element M.

\section{REFERENCES}

Berry, F. M., Joubert, C. E., \& Baumeister, A. A. Stimulus selection and meaningfulness at different stages of paired-associate learning. Journal of Experimental Psychology, 1972, 95, 189-194.

James, C. T., \& Greeno, J. G. Stimulus selection at different stages of paired-associate learning. Journal of Experimental Psychology, in press.

Siegel, S. Nonparametric statistics for the behavioral sciences. New York: McGraw-Hill, 1956.

Spear, N. E., Ekstrand, B. R., \& Underwood, B. J. Association by contiguity. Journal of Experimental Psychology, 1964, 67, 151-161.

Underwood, B. J., Ham, M., \& Ekstrand, B. Cue selection in paired-associate learning. Journal of Experimental Psychology, 1962, 64, 405-409.

(Received for publication December 31, 1972.) 